\title{
Postoperative Transient Reduced Diffusion in the Ipsilateral Striatum and Thalamus
}

K. Kamiya, N. Sato, Y. Nakata, K. Ito, Y. Kimura, M. Ota, A. Takahashi, H. Mori, A. Kunimatsu, and K. Ohtomo

\begin{abstract}
BACKGROUND AND PURPOSE: Restriction of diffusion has been reported in the early phase of secondary neuronal degeneration, such as Wallerian degeneration. The purpose of this study was to investigate postoperative transient reduced diffusion in the ipsilateral striatum and thalamus as a remote effect of surgery.
\end{abstract}

MATERIALS AND METHODS: Six hundred two postoperative MR imaging examinations in 125 patients after cerebral surgery were retrospectively reviewed, focusing on the presence of reduced diffusion in the striatum and/or thalamus. The distribution of reduced diffusion in the striatum was classified into 3 groups: anterior, central, and posterior. Reduced diffusion in the thalamus was also classified on the basis of the anatomic locations of the thalamic nuclei. Further follow-up MRI was available in all patients with postoperative reduced diffusion, and acute infarctions were excluded. The patient medical records were reviewed to evaluate neurologic status.

RESULTS: Restriction of diffusion was observed in the striatum and/or thalamus ipsilateral to the surgical site in 17 patients (13.6\%). The distribution of signal abnormality correlated with the location of the operation, in concordance with the architecture of the striatocortical and thalamocortical connections. Reduced diffusion was observed from days 7 to 46 after the operation, especially during days $8-21$. The signal abnormalities completely resolved on follow-up examinations. The median follow-up period was 202 days (interquartile range, 76-487 days).

CONCLUSIONS: Postoperative transient reduced diffusion in the ipsilateral striatum and/or thalamus likely represents an early phase of secondary neuronal degeneration based on its characteristic distribution and time course. Clinically, this reduced diffusion should not be mistaken for postoperative ischemic injury.

ABBREVIATIONS: GABA = gamma-aminobutyric acid; OFC = orbitofrontal cortex; $\mathrm{PFC}=$ prefrontal cortex; $r A D C=$ relative $A D C$ ratio; WHO $=$ World Health Organization

D WI is an imaging technique that measures the degree of water diffusion in vivo and has clinical utility, particularly in the early diagnosis of acute ischemia. ${ }^{1,2}$ Reduced diffusion can, however, be seen in any lesion that alters the motion of water molecules. DWI has been shown to be useful in the diagnosis of abscess, multiple sclerosis, epidermoid tumor, diffuse axonal injury, encephalitis, and prion disease. ${ }^{3,4}$ Restriction of water diffusion has also been described in the early phase of secondary neuronal de-

Received March 19, 2012; accepted after revision May 26.

From the Departments of Radiology (K.K., N.S., Y.N., K.I., Y.K.) and Neurosurgery (A.T.), National Center Hospital of Neurology and Psychiatry, Kodaira, Tokyo, Japan; Department of Mental Disorders (M.O.), Research National Institute of Neuroscience, National Center of Neurology and Psychiatry, Kodaira, Tokyo, Japan; and Department of Radiology (H.M., A.K., K.O.), Faculty of Medicine, University of Tokyo Hospital, Bunkyo-ku, Tokyo, Japan.

Please address correspondence to Noriko Sato, MD, Department of Radiology, National Center Hospital of Neurology and Psychiatry, 4-1-1 Ogawahigashi-chyo, Kodaira, Tokyo 187-8551 Japan; e-mail: snoriko@ncnp.go.jp

http://dx.doi.org/10.3174/ajnr.A3242 generation, such as Wallerian degeneration and transneuronal degeneration. ${ }^{5-15}$ Diffusion abnormality due to Wallerian degeneration is known to develop within as little as 2 days after injury ${ }^{12}$ and to last for at least 2 weeks. ${ }^{13}$ Although it has been well-documented that secondary degeneration occurs in the thalamus following ischemic infarction in the MCA territory, ${ }^{16-18}$ reports on MR imaging findings of secondary degeneration in the striatum have been very sparse. ${ }^{19}$ In addition, there have been no reports on the depiction of the early phase of postoperative secondary degeneration by using DWI.

In postoperative follow-up of brain surgeries, we have occasionally observed reduced diffusion in the ipsilateral striatum and/or thalamus, remote from the operative bed, and topographic correlations were suspected between the surgical site and the distribution of reduced diffusion. These signal abnormalities were not associated with any referable neurologic deficits at the time of MR imaging, and completely resolved on follow-up examinations. The purpose of this study was to describe postoperative transient reduced 


\section{Type1}

\section{Head of caudate and the most anterior part of putamen}

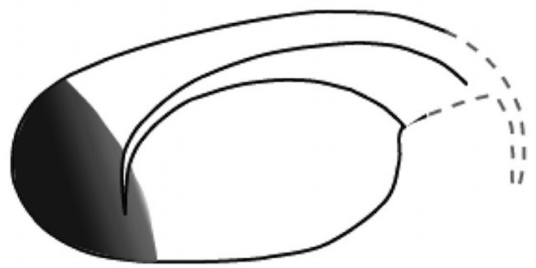

\section{Type2}

\section{Body of caudate and the central portion of putamen}

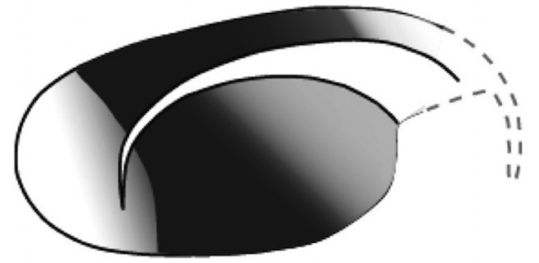

\section{Type3}

\section{Posterior putamen}

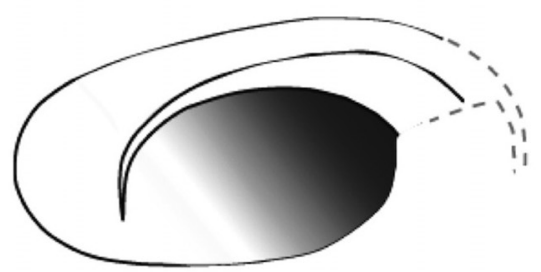

FIG 1. Classification of the distribution of reduced diffusion in the striatum.

diffusion in the ipsilateral striatum and thalamus, investigating the correlation between the surgical sites and the distribution of reduced diffusion, as well as the time course of signal changes.

\section{MATERIALS AND METHODS}

\section{Patients}

A retrospective review of radiologic records in 2 hospitals from January 2008 to April 2012 revealed 134 patients who underwent cerebral surgery between January 2008 and January 2012. Institutional review board approval was not required for studies involving retrospective image or case record review. The inclusion criteria were as follows: 1) pre- and postoperative DWI was available; and 2) when diffusion changes were present on postoperative scans, further follow-up MRI was ordered and was available. Simple callosotomy, biopsy, and surgeries for intraventricular or ex-
Table 1: Location of the operation and presence of postoperative reduced diffusion in the striatum and/or thalamus in 125 patients

\begin{tabular}{|c|c|c|c|c|}
\hline \multirow[b]{3}{*}{ Location of Operation } & \multicolumn{3}{|c|}{ Reduced Diffusion } & \multirow{2}{*}{$\begin{array}{l}\text { Total } \\
\text { No. of }\end{array}$} \\
\hline & Po & & & \\
\hline & \multicolumn{3}{|c|}{ Striatum Thalamus N } & Patients \\
\hline Frontal lobe ${ }^{a}$ & 9 & 2 & 35 & 44 \\
\hline Frontotemporal $^{a}$ & 2 & 1 & 3 & 5 \\
\hline Frontoparietal & 0 & 0 & 1 & 1 \\
\hline Temporal lobe & 0 & 0 & 47 & 47 \\
\hline Temporoparietal & 0 & 0 & 1 & 1 \\
\hline Temporo-occipital & 0 & 0 & 1 & 1 \\
\hline Parietal lobe & 0 & 0 & 11 & 11 \\
\hline Parieto-occipital & 0 & 0 & 3 & 3 \\
\hline Occipital lobe & 0 & 0 & 4 & 4 \\
\hline Hemispheric disconnection $^{a}$ & 2 & 2 & 0 & 2 \\
\hline Posterior disconnection ${ }^{a}$ & $1^{\mathrm{b}}$ & 4 & 1 & 5 \\
\hline Temporal disconnection & 0 & 0 & 1 & 1 \\
\hline Total & 14 & 9 & 108 & 125 \\
\hline
\end{tabular}

a Reduced diffusion was observed in both the striatum and thalamus in 2 patients with operations in the frontal lobe, 1 with an operation in the frontotemporal lobe, 2 with hemispheric disconnection, and 1 with posterior disconnection.

${ }^{\mathrm{b}}$ In this patient, disconnection involved the precentral gyrus.

tra-axial lesions were not reported in the 134 patients. Of the 134 patients, 9 additional patients who had direct involvement or injury of the striatum or thalamus due to either the original lesion or the surgical procedure ( 5 patients with glioblastoma, 1 with metastatic adenocarcinoma, 1 with Rasmussen encephalitis, 1 with hemimegalencephaly, and 1 with striatal hematoma) were excluded. Therefore, this study comprised 125 patients (56 females and 69 males; $0-87$ years of age; mean age, $38.0 \pm 22.0$ years) who underwent cerebral surgery. The distribution of the original lesion pathology was as follows: 23 low-grade brain tumors (WHO grades I and II), 50 high-grade brain tumors (WHO grades III and IV), 19 cases of focal cortical dysplasia, 1 case of hemimegalencephaly, 3 cortical tubers, 8 vascular malformations, 15 cases of hippocampal sclerosis, and 1 intracerebral hematoma. Specific histopathologic diagnosis could not be made in 5 patients who underwent epilepsy surgery, because the obtained specimens included only nonspecific gliosis and infiltration of inflammatory cells.

The median first follow-up MR imaging was 3 days (interquartile range, 2-7 days) after surgery, and all postoperative MRI was clinically indicated. Sixteen patients had only 1 postoperative MR imaging. Thirty-eight patients had 2 postoperative MRIs. The other 71 patients had serial postoperative MRIs, consisting of 3-20 examinations. Six hundred two postoperative MRI were evaluated in this study, and the median follow-up period was 202 days (interquartile range, 76-487 days). Five hundred twentyfour examinations were performed by using $1.5 \mathrm{~T}$ systems, whereas 78 examinations were performed by using $3 \mathrm{~T}$ systems. The patients' medical records were reviewed to evaluate their neurologic status at the time of MR imaging. MR imaging indications included follow-up to investigate residual or recurrent lesions, especially neoplasms that were not seen well-enough on CT. At least $1 \mathrm{MR}$ imaging was performed shortly after the operation on the basis of a clinical requirement to evaluate surgical complications, including infarctions. Some patients underwent several MRIs due to specific problems such as subdural hematoma/effusion, CSF leakage, or follow-up of malignancies. 


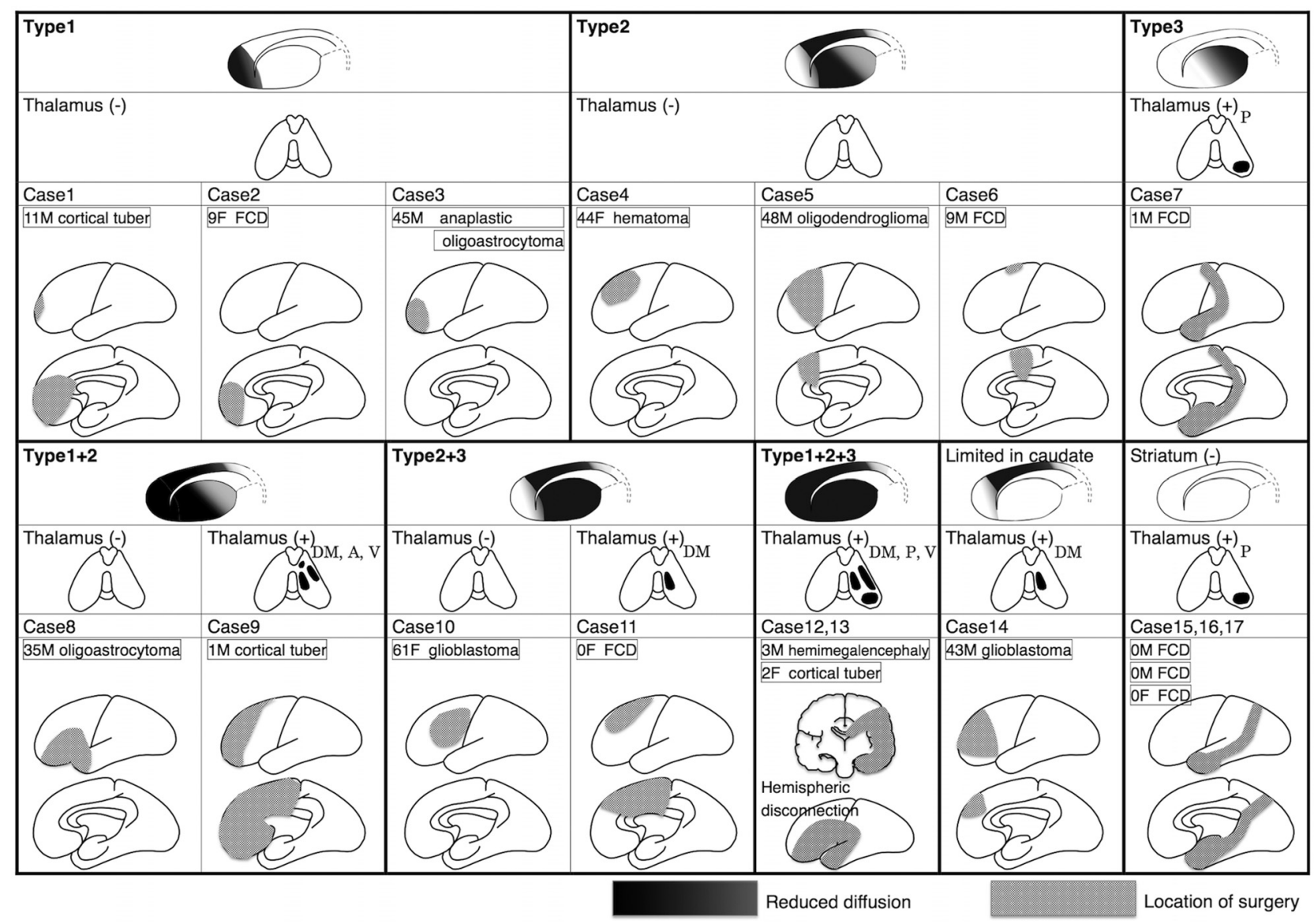

FIG 2. Schematic illustration demonstrating surgical sites and distributions of reduced diffusion in the striatum and thalamus in 17 cases. For simplification, all cases are depicted in the left hemisphere. A indicates anterior nuclear group; V, ventral nuclei; P, pulvinar; DM, dorsomedial nucleus; FCD, focal cortical dysplasia.
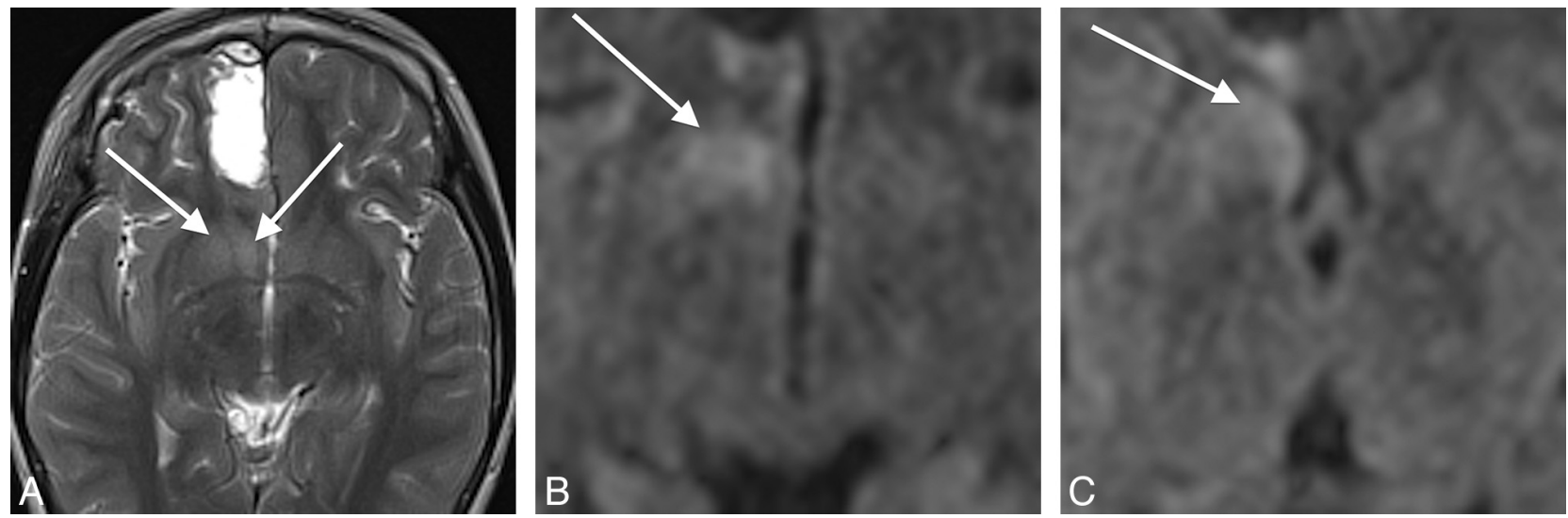

FIG 3. A 9-year-old girl who underwent resection of focal cortical dysplasia in the right frontal lobe (case 2). A, T2WI 10 days after surgery. The right OFC has been partially resected. The head of the caudate and the anterior end of the putamen look slightly hyperintense (arrows). B and C, DWI on day 10 shows increased signal intensity in the head of the caudate and the anterior end of the putamen (arrows). This case is classified as type 1.

\section{MR Imaging}

All patients underwent MR imaging by using 1.5T or 3T systems with varying imaging techniques. Single-shot echo-planar DWI was obtained by using the following parameters: TR, 3500-13,200 ms; TE, 53-95 ms; FOV, 21-23 × 21-23 cm; acquisition matrix, 96-128 $\times$ 96-128; section thickness, $3-5 \mathrm{~mm}$ with a gap of $0-2 \mathrm{~mm}$; b-values of 0 and $1000 \mathrm{~s} / \mathrm{mm}^{2}$; diffusion encoding gradient in 3-15 noncollinear directions; and NEX, 1-3, depending on the imaging system used. An ADC map was generated in each examination. T1WI (TR, 440-603 ms; TE, 9-12 ms; FOV, 21-23 × 20-21 cm; acquisition matrix, 240-320 $\times 169-256$; section thickness, 3-5 mm with a gap of 1-2 mm, and NEX, 1) and T2WI (TR, 3800-5176 ms; TE, 80-105 ms; FOV, 21-23 × 20-21 cm; acquisition matrix, 256-512 $\times 192-$ 348 ; section thickness, $3-5 \mathrm{~mm}$ with a gap of $0-2 \mathrm{~mm}$; and NEX, 1-2) were performed, along with other sequence images relevant to each case. 

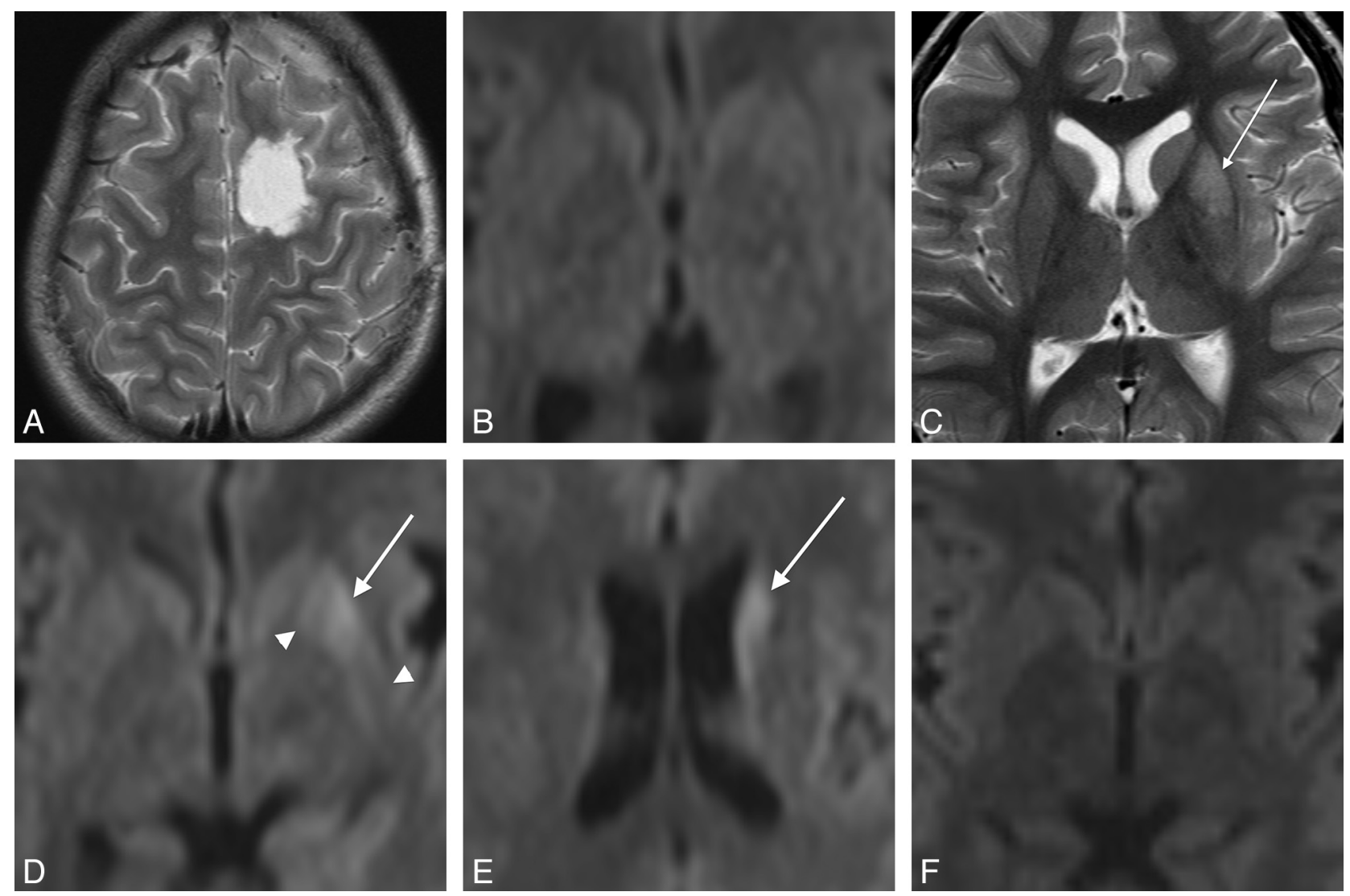

FIG 4. A 9-year-old boy who underwent resection of focal cortical dysplasia in the left frontal lobe (case 6). $A$, T2WI at day 1 . The left PFC has been partially resected. $B$, DWI on day 1 shows no signal abnormalities in the striatum or thalamus. C, T2WI on day 15 shows hyperintensity in the central portion of the putamen. $D$ and $E$, DWI on day 15 shows increased signal intensity in the body of the caudate and the central putamen (arrows). Note that the most anterior and posterior parts of the putamen are spared (white arrowheads). This case is classified as type 2. F, DWI on day 133 shows complete resolution of the signal abnormalities.

\section{Qualitative Image Analysis}

Image assessment was based on agreement between 2 neuroradiologists (K.K. and N.S., with 5 and 21 years of experience with MR images, respectively) who were blinded to the patient information, and the final decisions were reached by consensus. Observers assessed the presence of hyperintensity in the striatum and thalamus on DWI. Restriction of diffusion was determined on the basis of qualitative visual assessment of ADC maps. Diffusion restriction was defined as darker than the normal-appearing contralateral striatum/ thalamus on ADC maps. Wallerian and transneuronal degeneration demonstrate DWI hyperintensity in the early phase and may become atrophied in the chronic stage. ${ }^{12,17}$ Therefore, the patients whose striatum and/or thalamus became atrophied on follow-up MRI were not excluded as having infarctions if signal abnormalities completely resolved on both DWI and T2WI.

When restriction of diffusion was seen in the striatum, its distribution was categorized into 3 types as follows (Fig 1). "Type 1" was defined as restricted diffusion limited to the head of the caudate and the anterior end of the putamen, whereas "Type 3 " was restricted diffusion limited to the posterior part of the putamen. "Type 2" was defined as restricted diffusion in the body of the caudate and the central portion of the putamen, bridging the anterior to posterior, but sparing both ends. The distribution of reduced diffusion in the thalamus was also recorded on the basis of the anatomic location of thalamic nuclei (dorsomedial nucleus, ventral nuclei, pulvinar, and anterior nuclear group). The location of the operation was compared with the distribution of reduced diffusion. Visual assessment of T1WI and T2WI was performed to evaluate signal and morphologic changes of the striatum and thalamus. We also investigated the time course of diffusion changes and patient baseline characteristics related to diffusion restriction, including age, sex, type of disease treated, and timing of postoperative MRI.

\section{Quantitative Analysis}

We also performed region-of-interest analysis to determine ADC values of the striatum and thalamus in all patients. In the DWInegative striatum or thalamus, oval ROIs were placed in the center of the putamina and thalami bilaterally. In the DWI-positive striatum or thalamus, ROIs were placed in the striatum and/or thalamus in areas with the highest signal intensity on DWI and in the corresponding areas on the contralateral side. ROIs were also placed in corresponding locations on follow-up MRI. Manual region-of-interest placement was performed independently by 2 neuroradiologists (K.K. and N.S.) by using T2WI and DWI as a reference. Interobserver variability was evaluated by calculating the intraclass correlation coefficient for single measurements $(0-$ 0.20 , poor correlation; $0.21-0.40$, fair correlation; $0.41-0.60$, moderate correlation; $0.61-0.80$, good correlation; and $0.81-$ 

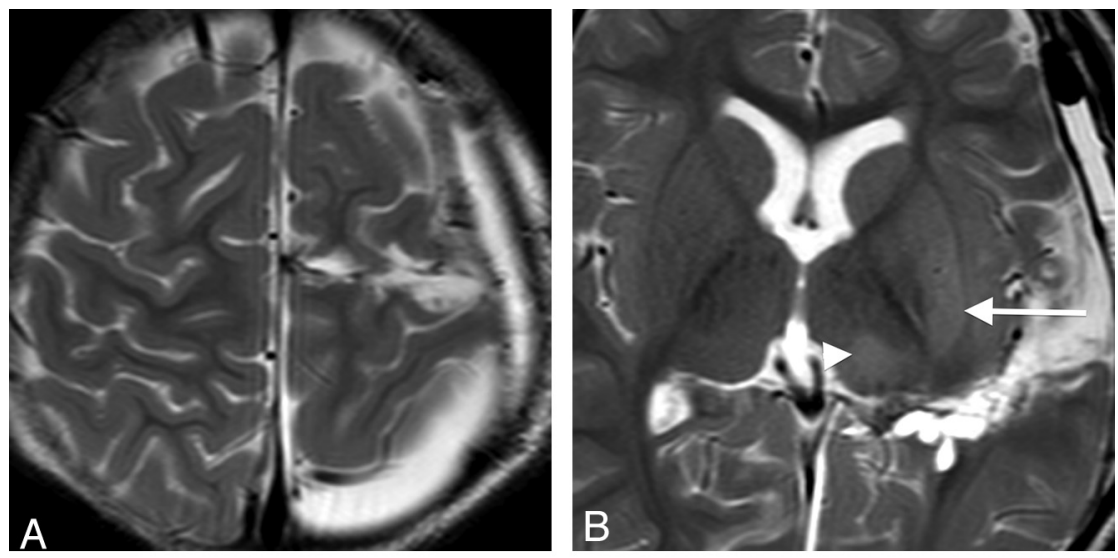

the 17 DWI-positive patients, signal abnormalities completely resolved on follow-up examinations and did not leave any tissue necrosis suggestive of infarction. None of these patients presented with acute neurologic deficits that were attributable to areas of reduced diffusion. No signal abnormality was observed in the contralateral striatum or thalamus.

\section{Topographic Correlations}

Forty-four, 47, 11, and 4 patients underwent surgery of the frontal, temporal, parietal, and occipital lobes, respectively (Table

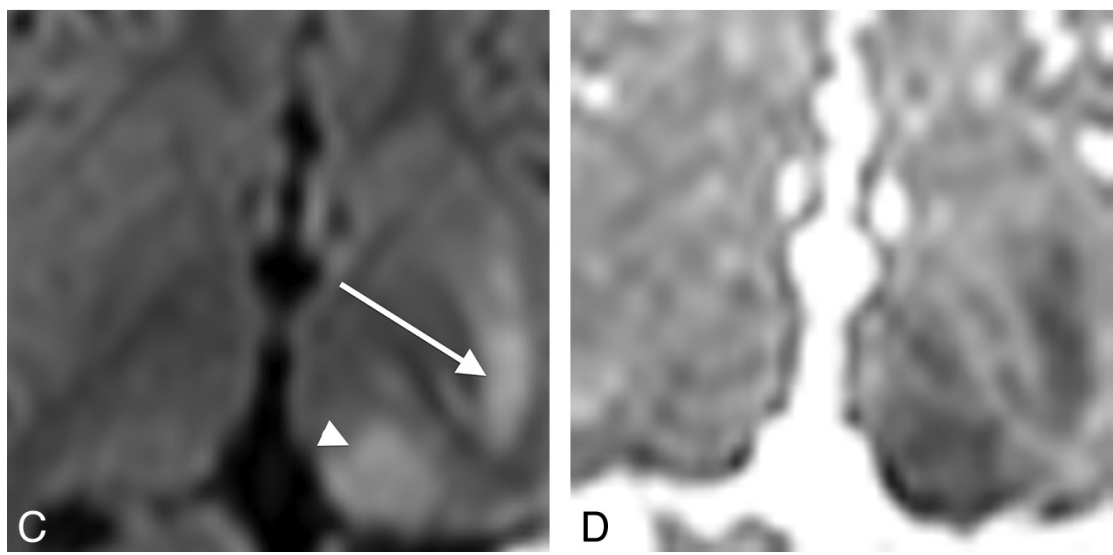
1). In 11 patients, resection involved $>2$ lobes. Two, 5, and 1 patient underwent hemispheric disconnection, posterior disconnection, and temporal disconnection, respectively. Reduced diffusion in the striatum was observed exclusively after surgery involving the frontal lobe (Table 1). Reduced diffusion in the thalamus was observed after surgery involving the frontal lobe or posterior disconnection.

The classification of signal abnormalities in the striatum demonstrated marked correlation with surgical locations. Figure 2 shows the locations of the operations along with the distribution of reduced diffusion in all $17 \mathrm{pa}$ tients who had reduced diffusion in the striatum and/or thalamus. Types 1, 2, and 3 were observed in patients after resection of the $\mathrm{OFC}, \mathrm{PFC}$, and motor/premotor cortices, reposterior disconnection surgery (case 7). $A$ and $B, T 2 W I$ on day 12 . Disconnection involves the left precentral gyrus. The posterior parts of the putamen (arrow) and pulvinar (arrowhead) show hyperintensity. $C$ and $D, D W I$ and the corresponding ADC map on day 12 show reduced diffusion in the posterior parts of the putamen (arrow) and pulvinar (arrowhead). This case is classified as type 3.

1.00, excellent correlation). Region-of-interest size was 20-30 $\mathrm{mm}^{2}$ for the striatum and $30-50 \mathrm{~mm}^{2}$ for the thalamus. rADC was calculated by the following formula: $\mathrm{ADC}=$ (average ADC value within the region of interest on surgical side)/(average ADC value within the region of interest on contralateral side). We focused on the postoperative period when DWI abnormalities were observed and compared rADC of the striatum and thalamus between DWIpositive and -negative patients. The rADC were also compared between these patients in the other periods when DWI abnormalities were absent on subjective image analysis. The Mann-Whitney $U$ test was used to investigate differences in rADC. The level of significance was defined at $P<.05$. All statistical analyses were performed by using the Statistical Package for the Social Sciences software, Version 11 (SPSS, Chicago, Illinois).

\section{RESULTS}

\section{Qualitative Analysis of Signal Changes}

Among 125 patients, 17 (13.6\%) showed reduced diffusion in the striatum and/or thalamus ipsilateral to the surgical site by qualitative assessment. Eight patients showed reduced diffusion limited to the striatum, while 3 patients showed reduced diffusion limited to the thalamus. Reduced diffusion was observed in both striatum and thalamus in 6 patients. The affected areas showed increased signal intensity on T2WI at the time of reduced diffusion. In all of spectively (Figs 3-5). Wider resection involving the OFC and PFC resulted in a combination of types 1 and 2 (Fig 6), whereas involvement of the PFC and motor/premotor cortices resulted in a combination of types 2 and 3. Reduced diffusion extending to the entire striatum (a combination of all 3 types) was observed after hemispheric disconnection. Only 1 case showed reduced diffusion that could not be classified into any of the 3 types or any combinations in which reduced diffusion was limited to the body of the caudate but absent in the putamen.

The distribution of signal abnormalities in the thalamus also depended on the location of the surgery. Reduced diffusion was limited to the pulvinar after posterior disconnection surgery (Fig 5), whereas it was also observed in the dorsomedial nucleus and ventral nuclei after hemispheric disconnection. Resection extending from the OFC to the PFC resulted in reduced diffusion in the dorsomedial nucleus, ventral nuclei, and anterior nuclear group, while the pulvinar was preserved (Fig 6). Reduced diffusion was limited to the dorsomedial nucleus after surgery in the PFC.

\section{Time Course of Signal and Morphologic Changes}

Reduced diffusion in the striatum or thalamus was found on days 7-46, especially during days 8-21 (Fig 7). Six patients had follow-up MRI on both days $0-6$ and days 7-46, and they presented a characteristic time course of diffusion changes; reduced diffu- 

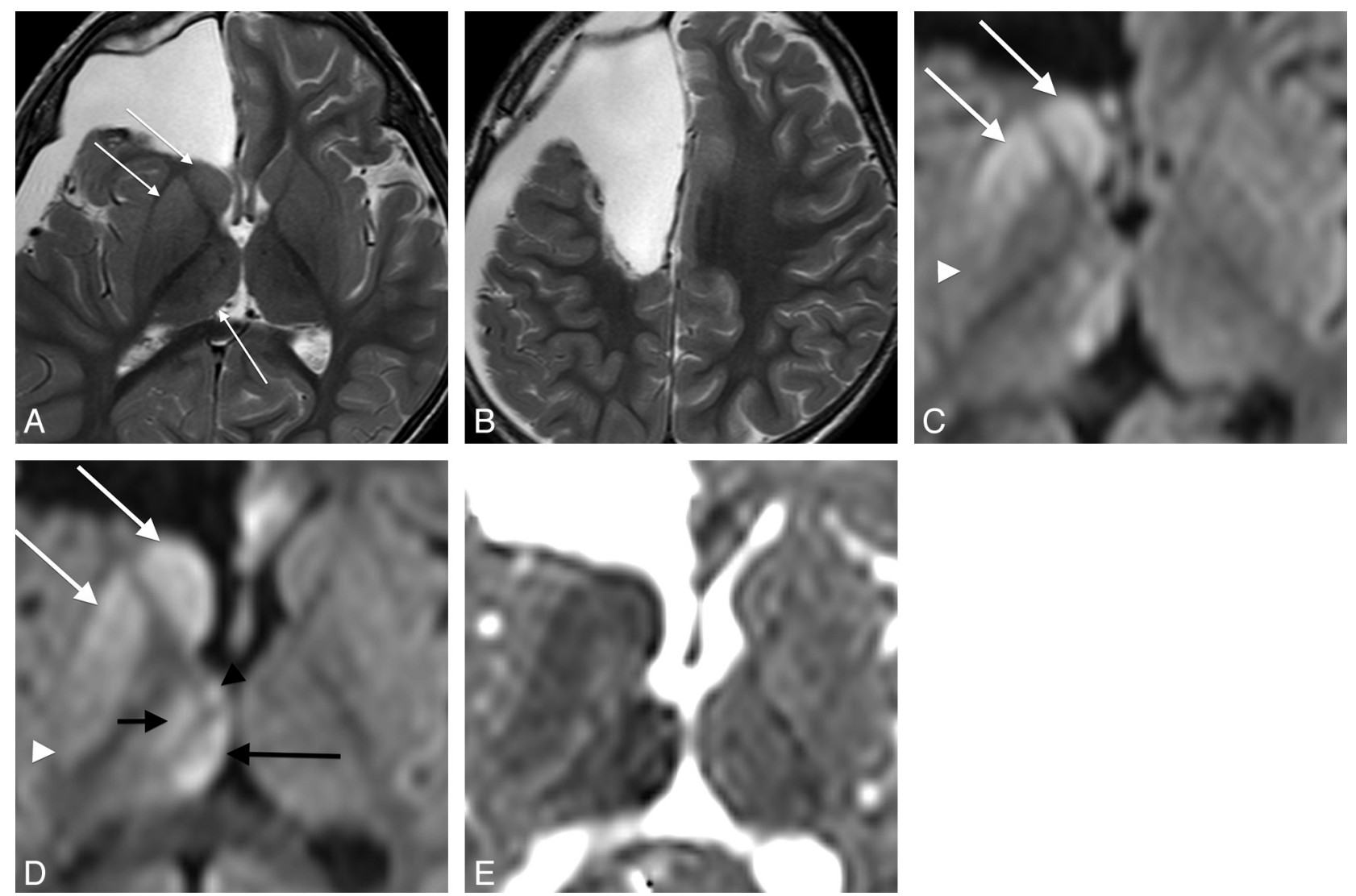

FIG 6. A 1-year-old boy with tuberous sclerosis who underwent resection of a cortical tuber in the right frontal lobe (case 9). A and B, T2WI on day 10. Surgical excision has extended from the right OFC to the PFC. The caudate head, the anterior putamen, and the pulvinar show slight hyperintensity (arrows). C and D, DWI on day 10. E, ADC map corresponding to $D$. Reduced diffusion is seen in the caudate head and body and in the putamen extending from the anterior end to the central portion (white arrows). Note that the most posterior part of the putamen is spared (white arrowhead). This case is classified as type $1+2$. In the thalamus, the dorsomedial nucleus (long black arrow), the ventral nuclei (short black arrow), and the anterior nuclei group (black arrowhead) show reduced diffusion.

sion was not present in the initial postoperative MRI on days $0-6$ but appeared on days 15-46 (Fig 4). Among them, 5 patients had reduced diffusion limited to the striatum. One patient had diffusion restriction in both the striatum and thalamus, and diffusion changes appeared at the same time. Diffusion restriction was observed on successive follow-up scans in 1 patient from days 7-16. The other 16 patients had only $1 \mathrm{MR}$ imaging with diffusion abnormalities during days 7-46, and their next MRI was performed after day 47.

None of the DWI-positive patients showed any morphologic changes of the affected striatum and/or thalamus, such as swelling or atrophy, at the time of reduced diffusion. In 7 patients, the affected striatum or thalamus or both became atrophied on further follow-up MRI on days 61-129.

\section{Baseline Characteristics of the Patients}

Table 2 summarizes the baseline characteristics of the patients. Because postoperative reduced diffusion presented here seemed to occur after operations involving the frontal and/or primary sensory cortices and posterior disconnection operations, we focused especially on those who underwent such operations, to investigate the differences between the DWI-positive and -negative cases. The presented diffusion abnormality seemed to occur more frequently in younger patients (most were younger than 10 years of age) who underwent surgery for intractable epilepsy. The median day of the first follow-up MR imaging in DWI-positive cases was day 9. In contrast, most DWI-negative patients were adults who underwent surgery for malignant neoplasms. Moreover, 40 of 108 DWI-negative patients did not have follow-up MRI during days 7-46.

\section{Quantitative Analysis of rADC}

The interobserver agreement for rADC measurements was excellent (intraclass correlation coefficient, 0.90 on the striatum and 0.84 on the thalamus). One hundred four MRIs were performed on days 7-46, when the diffusion restriction of the striatum and/or thalamus was observed. There were 16 and 9 MRIs with DWI abnormalities in the striatum and thalamus, respectively. The mean rADC for the 2 observers was significantly decreased on days 7-46 in both the striatum and thalamus in the DWI-positive cases, compared with the DWI-negative cases (Table 3). The patients who presented with reduced diffusion in the striatum during days 7-46 had 7 MRIs on days $0-6$ and 33 MRIs after day 47. The mean rADC of the striatum in these cases was $1.04(95 \%$ confidence interval, $0.96-1.12, n=7$ ) on days $0-6$ and 1.01 $(0.96-1.05, n=33)$ after day 47. Significant differences in rADC were not observed in these periods, compared with DWI-negative cases (days $0-6,1.01$ [0.99-1.03, $n=81]$; day 47 and after, 0.99 $[0.98-1.00, n=377])$. The patients who presented with reduced diffusion in the thalamus during days 7-46 had only 2 MRIs on days $0-6$ and 23 MRIs after day 47. The mean rADC of the thalamus in these patients was 1.05 (95\% confidence interval not 


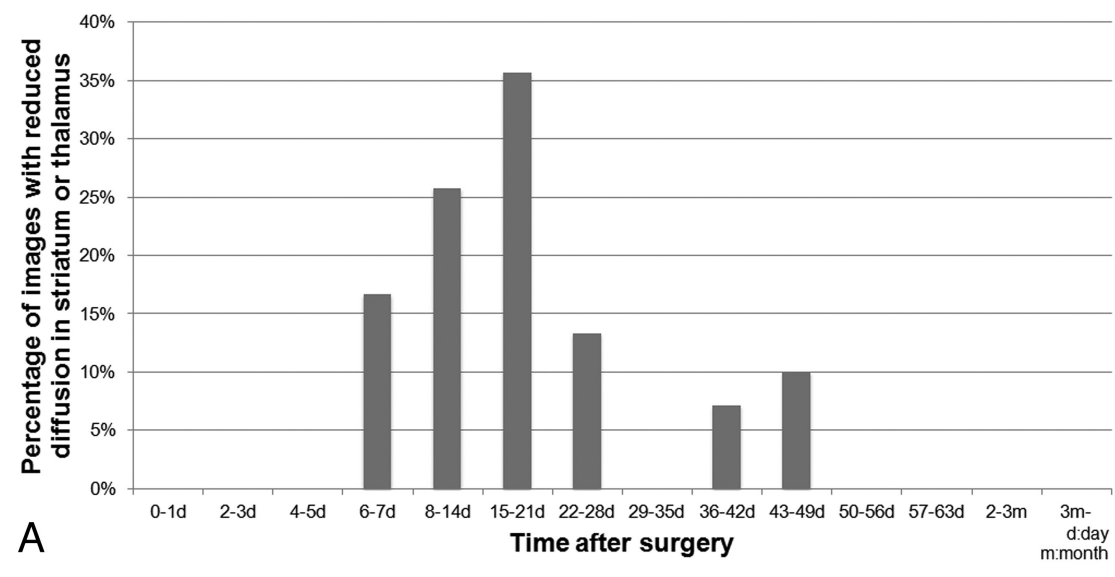

strength of our study is the more precise anatomic correlation between damaged cortical areas and distribution of striatal signal abnormalities based on the assessment of a larger number of cases. In addition, previous reports of secondary thalamic degeneration in humans focused on T2WI, ${ }^{16,17}$ not on DWI, though reduced diffusion in the early phase of secondary thalamic degeneration has been reported in rat models of MCA infarction. ${ }^{11}$ In our cases of postoperative secondary degeneration, reduced diffusion was not observed on days $0-6$ but appeared later on days 7-46. Secondary degeneration, including Wallerian degeneration and transneuronal degeneration, demonstrates DWI hyperintensity in the early phase and may become atrophied in the chronic stage. ${ }^{12,17}$ Atrophy of the affected striatum and/or thalamus was observed after days 61-129 in some of our cases.

Most interesting, reduced diffusion in the striatum was observed exclusively after surgery involving the frontal lobe. This seems meaningful because the striatum receives input principally from the frontal cortex. Moreover, the distribution of signal abnormality correlated with the surgical location and was consistent with the anatomic architecture of striatocortical and thalamocortical connections described below.

The frontostriatal projection is topoapplicable, $n=2)$ on days $0-6$ and $1.00(0.96-1.04, n=23)$ after day 47. Significant differences in rADC were not observed in these periods, compared with DWI-negative cases (days 0-6, 1.02 [1.00-1.03, $n=86]$; day 47 and after, 1.02 [1.01-1.02, $n=387])$.

\section{DISCUSSION}

The restriction of water diffusion can be seen in the early phase of secondary neuronal degeneration. ${ }^{5-15}$ We report postoperative transient reduced diffusion in the ipsilateral striatum and/or thalamus, with diffusion restriction observed on days 7-46 (mostly days 8-21), and a marked correlation between the surgical location and the distribution of signal abnormalities within the striatum and/or thalamus. This correlation is consistent with the anatomy of neuronal connectivities and suggests that the observed reduced diffusion represents the early phase of secondary neuronal degeneration. Our present observations may help strengthen our knowledge of striatocortical and thalamocortical connections and will prevent postoperative MR imaging findings from being misinterpreted as acute infarction.

There has been 1 previous report describing findings of secondary striatal degeneration on DWI. ${ }^{19}$ Similar to our results, the striatum in this report showed reduced diffusion 8-60 days after external capsular hemorrhage in 9 patients of 28, presumably representing the early phase of secondary degeneration. ${ }^{19}$ The graphically organized in a ventromedial-to-dorsolateral gradient, from limbic and cognitive to motor functions. ${ }^{20}$ The ventromedial striatum receives its frontal input from the medial and lateral OFC, anterior cingulate cortex, and agranular insular cortex. ${ }^{21-23}$ The dorsolateral prefrontal cortex projects to the central portion of the striatum, bridging the ventral and dorsal areas, ${ }^{24,25}$ whereas the dorsal striatum receives input mainly from the motor/premotor cortices. ${ }^{26-28}$ Recent studies by using fMRI, FDG-PET, and DTI have provided in vivo evidence that supports this connectivity-based parcellation of the striatum. ${ }^{29-34}$ In this study, damage to the OFC, PFC, and motor/premotor cortices caused reduced diffusion in the anterior, central, and posterior portion of the striatum (types 1, 2, and 3), respectively. These results were consistent with the anatomy of striatocortical connections and strongly suggested that the reduced diffusion observed here represented the early phase of secondary neuronal degeneration.

Only 1 patient showed reduced diffusion limited to the caudate body, sparing the putamen. This case could not be classified into any of the 3 types, though it seemed to be a variant of type 2, considering the distribution of reduced diffusion and the location of surgery. This case may represent a smaller subdivision of the striatocortical connections. Although the caudate body and the central putamen were dealt with together as type 2 in this study, the cortices from which 
Table 2: Baseline characteristics of patients with and without reduced diffusion in the striatum and/or thalamus

\begin{tabular}{|c|c|c|c|}
\hline \multirow[b]{3}{*}{ Location of Operation } & \multicolumn{3}{|c|}{ Reduced Diffusion } \\
\hline & Positive & Negative & \\
\hline & $\begin{array}{l}\text { Frontal Lobe or } \\
\text { Primary Sensory Cortices, } \\
\text { or Posterior Disconnection }\end{array}$ & $\begin{array}{l}\text { Frontal Lobe or } \\
\text { Primary Sensory Cortices, } \\
\text { or Posterior Disconnection }\end{array}$ & Others \\
\hline No. of patients (M/F) & $17(11: 6)$ & $43(28: 15)$ & $65(30: 35)$ \\
\hline Age (mean) (yr) & $18.4 \pm 21.8$ & $40.7 \pm 19.1$ & $41.3 \pm 21.4$ \\
\hline Total No. of MRIs (1.5T/3T) & $64(40 / 24)$ & $286(262 / 24)$ & $252(222 / 30)$ \\
\hline \multicolumn{4}{|l|}{ No. of MRIs in each period } \\
\hline Days 0-6 & 7 & 34 & 47 \\
\hline Days 7-46 (days 8-21) & $20(14)$ & $36(9)$ & $48(22)$ \\
\hline Day 47 and after & 37 & 216 & 157 \\
\hline Median time of the first follow-up MRI (interquartile range) & Day 9 (3-12) & Day $3(2-5)$ & Day $3(2-7)$ \\
\hline \multicolumn{4}{|l|}{ Type of disease } \\
\hline Low-grade tumor ${ }^{a}$ & 2 & 10 & 11 \\
\hline High-grade tumor ${ }^{\mathrm{b}}$ & 3 & 21 & 26 \\
\hline Focal cortical dysplasia & 7 & 5 & 7 \\
\hline Hemimegalencephaly & 1 & 0 & 0 \\
\hline Cortical tuber & 3 & 0 & 0 \\
\hline Vascular malformation & 0 & 4 & 4 \\
\hline Hippocampal sclerosis & 0 & 1 & 14 \\
\hline Hematoma & 1 & 0 & 0 \\
\hline Gliosis & 0 & 2 & 3 \\
\hline
\end{tabular}

${ }^{a} \mathrm{WHO}$ grades I and II.

b WHO grades III and IV.

Table 3: Relationship between DWI abnormality and rADC on days $7-46$

\begin{tabular}{llll}
\hline Location & Reduced Diffusion & $\begin{array}{c}\text { Mean rADC } \\
(95 \% \mathrm{Cl})\end{array}$ & $\boldsymbol{P}$ Value \\
\hline Striatum & Positive $(n=16)$ & $0.76(0.73-0.79)$ & $<.0001$ \\
& Negative $(n=88)$ & $1.02(1.00-1.04)$ & \\
Thalamus & Positive $(n=9)$ & $0.71(0.66-0.76)$ & $<.0001$ \\
& Negative $(n=95)$ & $1.00(0.98-1.01)$ & \\
\hline
\end{tabular}

Note:- $\mathrm{Cl}$ indicates confidence interval.

${ }^{a} \mathrm{rADC}=$ (average $A D C$ value within the $\mathrm{ROI}$ on surgical side)/(average ADC value within the ROI on contralateral side).

they primarily receive input may be slightly different. The other explanation is that the time course of reduced diffusion may be slightly different between the caudate and putamen.

As for the thalamus, the dorsomedial nucleus, the ventral anterior and ventral lateral nuclei, ventral posterior nucleus, and the anterior nuclear group are connected to the PFC, premotor/motor cortex, primary sensory cortex, and anterior cingulum, respectively. The pulvinar is connected to the association cortex of the occipital, parietal, and posterior temporal lobes. ${ }^{35,36}$ Therefore, damage to these cortical areas and thalamic radiations may cause secondary degeneration in the corresponding thalamic nuclei. ${ }^{16,17}$ In this study, reduced diffusion in the thalamus occurred when a sufficient amount of central tissue containing thalamic radiation was damaged. Particularly, damage to the posterior thalamic radiations and the PFC resulted in degeneration of the pulvinar and dorsomedial nucleus, respectively.

The observed postoperative diffusion restriction in the striatum and/or thalamus may occur more frequently in younger patients, perhaps related to the developing brain or to epilepsy surgery. It may be related to increased water content or reduced myelination, as has been speculated in previous reports that observed early Wallerian degeneration on DWI more frequently in the neonatal brain. ${ }^{12,14,15}$ Otherwise, it may be due to specific features of neuronal transmission in the developing brain, including immaturity of glutamate receptors ${ }^{37-39}$ and GABA receptor signaling, ${ }^{40,41}$ leading to vulnerability to glutamate-mediated excitotoxicity. Alterations of glutamate receptor function and GABAergic signaling have been described in epilepsy, ${ }^{40-42}$ as well.

There are some limitations to our study. First, a considerable number of DWI-negative patients did not have follow-up MRI on days 7-46. In surgery for malignant neoplasm, the first postoperative MR imaging is usually performed within $48-72$ hours of the operation to avoid contrast enhancement of the surgical margin that complicates the evaluation of residual tumor. ${ }^{43}$ Therefore, the diffusion changes may have been missed in these patients. Second, histopathologic correlation with diffusion abnormality was lacking. Further research, including animal experiments and functional imaging of blood perfusion and/or metabolism, will be helpful in widening our knowledge of the mechanism of reduced diffusion. Last, this study is limited by its retrospective nature, making it more challenging to assess the clinical relevance of the diffusion restriction observed.

\section{CONCLUSIONS}

In this study, we described the presence and time course of postoperative transient reduced diffusion in the ipsilateral striatum and thalamus. We correlated the distribution of signal abnormalities with the surgical location and found that this was consistent with the anatomy of striatocortical and thalamocortical connections. The characteristic distribution and time course of signal abnormalities suggest that the reduced diffusion observed here represents the early phase of secondary neuronal degeneration. In clinical practice, postoperative transient reduced diffusion in the ipsilateral striatum and/or thalamus should not be mistaken for other postoperative lesions such as acute ischemia. 
Disclosures: Akira Kunimatsu—UNRELATED: Grant: research grant from Bayer Healthcare. * *Money paid to the institution.

\section{REFERENCES}

1. Sorensen AG, Buonanno FS, Gonzalez RG, et al. Hyperacute stroke: evaluation with combined multisection diffusion-weighted and hemodynamically weighted echo-planar MR imaging. Radiology 1996;199:391-401

2. Lövblad KO, Laubach HJ, Baird AE, et al. Clinical experience with diffusion-weighted MR in patients with acute stroke. AJNR Am J Neuroradiol 1998;19:1061-66

3. Mukherji SK, Chenevert TL, Castillo M. Diffusion-weighted magnetic resonance imaging. J Neuroophthalmol 2002;22:118-22

4. Young GS, Geschwind MD, Fischbein NJ, et al. Diffusion-weighted and fluid-attenuated inversion recovery imaging in Creutzfeldt-Jakob disease: high sensitivity and specificity for diagnosis. AJNR Am J Neuroradiol 2005;26:1551-62

5. Nakane M, Tamura A, Miyasaka N, et al. Astrocytic swelling in the ipsilateral substantia nigra after occlusion of the middle cerebral artery in rats. AJNR Am J Neuroradiol 2001;22:660-63

6. Castillo M, Mukheriji SK. Early abnormalities related to postinfarction Wallerian degeneration: evaluation with MR diffusionweighted imaging. J Comput Assist Tomogr 1999;23:1004-07

7. Kang DW, Chu K, Yoon BW, et al. Diffusion-weighted imaging in Wallerian degeneration. J Neurol Sci 2000;178:167-69

8. Kinoshita T, Moritani T, Shrier DA, et al. Secondary degeneration of the substantia nigra and corticospinal tract after hemorrhagic middle cerebral artery infarction: diffusion-weighted MR findings. Magn Reson Med Sci 2002;1:175-78

9. Pierpaoli C, Barnett A, Pajevic S, et al. Water diffusion changes in Wallerian degeneration and their dependence on white matter architecture. Neuroimage 2001;13:1174-85

10. Zhao F, Kuroiwa T, Miyasaka N, et al. Characteristic changes in $\mathrm{T}(2)$-value, apparent diffusion coefficient, and ultrastructure of substantia nigra evolving exofocal postischemic neuronal death in rats. Brain Res 2001;895:238-44

11. Abe $\mathrm{O}$, Nakane M, Aoki S, et al. MR imaging of postischemic neuronal death in the substantia nigra and thalamus following middle cerebral artery occlusion in rats. NMR Biomed 2003;16:152-59

12. Mazumdar A, Mukherjee P, Miller JH, et al. Diffusion-weighted imaging of acute corticospinal injury preceding Wallerian degeneration in the maturing human brain. AJNR Am J Neuroradiol 2003;24:1057-66

13. Uchino A, Sawada A, Takase Y, et al. Transient detection of early Wallerian degeneration on diffusion-weighted MRI after an acute cerebrovascular accident. Neuroradiology 2004;46:183-88

14. Kirton A, Shroff M, Visvanathan T, et al. Quantified corticospinal tract diffusion restriction predicts neonatal stroke outcome. Stroke 2007;38:974-80

15. Domi T, deVeber G, Shroff M, et al. Corticospinal tract pre-Wallerian degeneration: a novel outcome predictor for pediatric stroke on acute MRI. Stroke 2009;40:780-87

16. Ogawa T, Yoshida Y, Okudera T, et al. Secondary thalamic degeneration after cerebral infarction in the middle cerebral artery distribution: evaluation with MR imaging. Radiology 1997;204:255-62

17. Nakane M, Tamura A, Sasaki Y, et al. MRI of secondary changes in the thalamus following a cerebral infarct. Neuroradiology 2002;44: 915-20

18. Nakane M, Tamura A, Nagaoka T, et al. MR detection of secondary changes remote from ischemia: preliminary observations after occlusion of the middle cerebral artery in rats. AJNR Am J Neuroradiol 1997; 18:945-50

19. Moon WJ, Na DG, Kim SS, et al. Diffusion abnormality of deep gray matter in external capsular hemorrhage. AJNR Am J Neuroradiol 2005;26:229-35

20. Haber SN, Fudge JL, McFarland NR. Striatonigrostriatal pathways in primates form an ascending spiral from the shell to the dorsolateral striatum. J Neurosci 2000;20:2369-82
21. Kunishio K, Haber SN. Primate cingulostriatal projection: limbic striatal versus sensorimotor striatal input. J Comp Neurol 1994;350:337-56

22. Haber SN, Kunishio K, Mizobuchi M, et al. The orbital and medial prefrontal circuit through the primate basal ganglia. J Neurosci 1995; 15:4851-67

23. Chikama M, McFarland N, Amaral DG, et al. Insular cortical projections to functional regions of the striatum correlate with cortical cytoarchitectonic organization in the primate. J Neurosci 1997; 17:9686-705

24. Selemon LD, Goldman-Rakic PS. Longitudinal topography and interdigitation of corticostriatal projections in the rhesus monkey. J Neurosci 1985;5:776-94

25. Yeterian EH, Pandya DN. Prefrontostriatal connections in relation to cortical architectonic organization in rhesus monkeys. J Comp Neurol 1991;312:43-67

26. Künzle $\mathrm{H}$. Bilateral projections from precentral motor cortex to the putamen and other parts of the basal ganglia: an autoradiographic study in Macaca fascicularis. Brain Res 1975;88:195-209

27. Künzle $H$. An autoradiographic analysis of the efferent connections from premotor and adjacent prefrontal regions (areas 6 and 9) in Macaca fascicularis. Brain Behav Evol 1978;15:185-234

28. Alexander GE, DeLong MR, Strick PL. Parallel organization of functionally segregated circuits linking basal ganglia and cortex. Annu Rev Neurosci 1986;9:357-81

29. Postuma RB, Dagher A. Basal ganglia functional connectivity based on a meta-analysis of 126 positron emission tomography and functional magnetic resonance imaging publications. Cereb Cortex 2006;16:1508-21

30. Di Martino A, Scheres A, Margulies DS, et al. Functional connectivity of human striatum: a resting state FMRI study. Cereb Cortex 2008; $18: 2735-47$

31. Bohanna I, Georgiou-Karistianis N, Egan GF. Connectivity-based segmentation of the striatum in Huntington's disease: vulnerability of motor pathways. Neurobiol Dis 2011;42:475-81

32. Draganski B, Kherif F, Klöppel S, et al. Evidence for segregated and integrative connectivity patterns in the human basal ganglia. $\mathrm{J} \mathrm{Neu-}$ rosci 2008;28:7143-52

33. Croxson PL, Johansen-Berg H, Behrens TE, et al. Quantitative investigation of connections of the prefrontal cortex in the human and macaque using probabilistic diffusion tractography. J Neurosci 2005;25:8854-66

34. Lehéricy S, Ducros M, Van de Moortele PF, et al. Diffusion tensor fiber tracking shows distinct corticostriatal circuits in humans. Ann Neurol 2004;55:522-29

35. Nieuwenhuys R, Voogd J, van Huijzen C. Human Central Nervous System. 3rd ed. New York: Springer-Verlag; 1988:237-46

36. Behrens TE, Johansen-Berg H, Woolrich MW, et al. Non-invasive mapping of connections between human thalamus and cortex using diffusion imaging. Nat Neurosci 2003;6:750-57

37. Johnston MV. Neurotransmitters and vulnerability of the developing brain. Brain Dev 1995; 17:301-06

38. Pellegrini-Giampietro DE, Gorter JA, Bennett MV, et al. The GluR2 (GluR-B) hypothesis: $\mathrm{Ca}(2+)$-permeable AMPA receptors in neurological disorders. Trends Neurosci 1997;20:464-70

39. Pellegrini-Giampietro DE, Bennett MV, Zukin RS. Are Ca(2+)-permeable kainate/AMPA receptors more abundant in immature brain? Neurosci Lett 1992;144:65-69

40. Galanopoulou AS. GABA(A) receptors in normal development and seizures: friends or foes? Curr Neuropharmacol 2008;6:1-20

41. Briggs SW, Galanopoulou AS. Altered GABA signaling in early life epilepsies. Neural Plast 2011;2011;527605

42. Chapman AG. Glutamate receptors in epilepsy. Prog Brain Res 1998; 116:371-83

43. Sato N, Bronen RA, Sze G, et al. Postoperative changes in the brain: MR imaging findings in patients without neoplasms. Radiology 1997;204:839-46 